\title{
Possible Origin of Aldosteronoma from Adrenohepatic Fusion Resulting in Intrahepatic Tumor ${ }^{*}$
}

\author{
Takumi Takeuchi ${ }^{1}$, Takashi Inoue ${ }^{2}$, Keiko Kanemoto ${ }^{2}$, Kazuchika Hagiwara ${ }^{3}$, Koji Mikami ${ }^{1}$, \\ Mami Hattori $^{1}$, Masayoshi Zaitsu ${ }^{1}$, Akiko Tonooka ${ }^{4}$, Toshimasa Uekusa ${ }^{4}$, Susumu Uda ${ }^{2}$ \\ ${ }^{1}$ Department of Urology, Kanto Rosai Hospital, Kawasaki, Japan \\ ${ }^{2}$ Nephrology, Kanto Rosai Hospital, Kawasaki, Japan \\ ${ }^{3}$ Diagnostic Radiology, Kanto Rosai Hospital, Kawasaki, Japan \\ ${ }^{4}$ Pathology, Kanto Rosai Hospital, Kawasaki, Japan \\ Email: takeuchit@abelia.ocn.ne.jp
}

Received August 9, 2013; revised August 28, 2013; accepted September 3, 2013

Copyright (C) 2013 Takumi Takeuchi et al. This is an open access article distributed under the Creative Commons Attribution License, which permits unrestricted use, distribution, and reproduction in any medium, provided the original work is properly cited.

\begin{abstract}
A 69-year-old woman was diagnosed with primary aldosteronism. An enhanced computed tomography (CT) scan before surgery indicated a right adrenal tumor outside the liver. Venous sampling tests revealed unilateral overproduction of aldosterone by the right adrenal gland. Separation of the right adrenal cortex from the liver parenchyma was impractical during a laparoscopic right adrenalectomy because of the solid attachment between the two. Therefore, the existence of adrenohepatic fusion was determined. An incision was made within the right adrenal gland, leaving completely the intrahepatic adrenal tissue on the inner side of the liver, because a partial hepatectomy was not preoperatively planned, and the patient was not informed of the consent before the surgery. Pathological examination did not reveal macro- or micro-adenomas in the resected right adrenal tissue. Aldosterone to renin ratio was as high as 1380 at 22 days following the surgery. Therefore, aldosteronoma originated from the adrenohepatic fusion that remained on the inner side of the liver was highly suspected. The patient's blood pressure was well controlled, and she did not prefer hepatectomy to be further performed, and therefore, medical therapy was continued. When planning the type of surgery (laparoscopic or open) in these potentially confusing cases, it might be necessary to consider a possibility of the unexpected intraoperative diagnosis and the immediate measures to be performed based on the diagnosis.
\end{abstract}

Keywords: Adrenal; Tumor; Adrenohepatic Fusion

\section{Introduction}

Primary aldosteronism is more frequent than previously speculated and it results in damaging the heart, blood vessels, and kidneys [1]. Majority of the hypertensive patients with primary aldosteronism, characterized by an autonomous secretion of aldosterone from the aldosteronoma, are diagnosed with the introduction of advanced imaging, such as computed tomography (CT) scans, magnetic resonance imaging (MRI), and ultrasound, as well as sophisticated advancements in endocrinology tests. Primary aldosteronism among hypertensive patients is determined by the aldosterone to renin ratio, and then confirmed by four testing procedures: oral sodium loading, saline infusion, fludrocortisone suppression, and captopril challenge. Furthermore, the laterality of aldosteronomas is determined by interventional venous sampling

*The authors declared no conflicts of interest. techniques $[2,3]$.

Those patients recently undergo laparoscopic adrenalectomy for antihypertensive drugs to be discontinued or reduced and for the vital organs such as the brain, heart, kidneys, and vessels to be protected from further damages [4]. Here, we present a case of primary aldosteronism, where adrenohepatic fusion was identified during a laparoscopic right adrenalectomy, and the aldosteronoma was speculated to be originated from adrenohepatic fusion.

\section{Case Report}

A 69-year-old woman was diagnosed with primary aldosteronism using endocrinology evaluation, following the cessation of antihypertensive drugs. The patient's serum potassium level was $3.1 \mathrm{mEq} / \mathrm{L}$, and the aldosterone to renin ratio was 406. After captopril challenge, the aldosterone to renin ratio was 249 (positive if $>200$ ) con- 
firming autonomous aldosterone production; however, after saline infusion, the plasma aldosterone concentration was $52.8 \mathrm{pg} / \mathrm{mL}$ (positive if $>60$ ). Venous sampling tests revealed unilateral overproduction of aldosterone by the right adrenal gland (Table 1).

During the first hospital visit before nine years, the patient had hypertension with hypokalemia $(2.8 \mathrm{mEq} / \mathrm{L})$ and a high aldosterone to renin ratio $(>200)$. Thereafter, hypertension and hypokalemia had been well controlled with the administration of angiotensin converting enzyme inhibitor combined with angiotensin receptor blocker. The patient suffered a cerebral infarction before a year.

An enhanced CT scan before the surgery revealed a right adrenal tumor responsible for the endocrine disorder located outside the liver; furthermore, liver cysts of various sizes were observed (Figure 1). A small liver cyst adjacent to the right adrenal mass altered the appropriate radiological diagnosis, because it displaced the adrenal mass located on the lateral inner side of the liver, and resembled an extrahepatic mass.

Finally, a laparoscopic right adrenalectomy was planned. The right adrenal vein branching from the inferior vena cava was identified and resected. During the surgery, the right adrenal cortex was solidly attached to the liver parenchyma, and separating the two that determined the existence of adrenohepatic fusion became impractical (Figure 2(a)). An incision was made within the right adrenal gland, leaving completely the intrahepatic adrenal tissue on the inner side of the liver (Figure 2(b)), because partial hepatectomy was not preoperatively planned and the patient was not informed of the consent related to the surgical procedure. In addition, the possibility remained that macro- or micro-adenomas existed in the resected right adrenal tissue (Figure 3). Postoperative course was uneventful.

Pathological examination did not reveal macro- or even micro-adenomas in the resected right adrenal tissue. Aldosterone to renin ratio was as high as 1380 at 22 days following the surgery, while serum potassium level was normalized to $4.5 \mathrm{mEq} / \mathrm{l}$, without the administration of eplerenone. Therefore, it was highly suspected that the aldosteronoma originated from the adrenohepatic fusion remained on the inner side of the liver.

\section{Discussion}

Adrenohepatic fusion is reported as the fusion of adrenal gland with liver, caused by the mesenchymal defect leading to the retardation of capsule formation with parenchymal mixing and the failure of local differentiation into fetal and later adult fat cells [5]. Consequently, no physical separation between the liver and the adrenal gland was noticeable. Furthermore, it is observed in nonhuman mammals [6,7].

Adrenohepatic fusion can be a hematogenous route of right adrenal metastasis from hepatocellular carcinoma [8-10]. Cortical adenomas arising in adrenohepatic fusion are difficult to distinguish from primary hepatic tumors using CT scans $[11,12]$; therefore, partial hepatectomy is often performed with the diagnosis of a malignant hepatocellular carcinoma. The location of adrenal ectopic or rest tissue can occur anywhere along the course of gonadal descent during embryogenesis. This essentially harmless tissue becomes hyperplastic or even tumorous in patients with primary or secondary adrenal pathology. For example, patients with congenital adrenal hyperpla-

Table 1. Venous sampling data.

\begin{tabular}{|c|c|c|c|}
\hline Before ACTH loading & Rt. adrenal vein & Lt. adrenal vein & IVC \\
\hline PAC & 586 & 54.1 & 39.4 \\
\hline $\mathrm{C}$ & 14.3 & 19.8 & 10.4 \\
\hline AV/IVC cortisol ratio & 1.3 & 1.9 & \\
\hline $\mathrm{PAC} / \mathrm{C}$ ratio & 41.0 & 2.73 & 3.79 \\
\hline $\mathrm{PAC} / \mathrm{C}$ lateralization ratio & 15.0 & & \\
\hline After ACTH loading & Rt. adrenal vein & Lt. adrenal vein & IVC \\
\hline PAC & 19,100 & 1620 & 298 \\
\hline $\mathrm{C}$ & 304 & 463 & 21.8 \\
\hline $\mathrm{AV} / \mathrm{IVC}$ cortisol ratio & 13.9 & 21.2 & \\
\hline $\mathrm{PAC} / \mathrm{C}$ ratio & 62.8 & 3.49 & 13.6 \\
\hline $\mathrm{PAC} / \mathrm{C}$ lateralization ratio & 18.0 & & \\
\hline
\end{tabular}

Rt.: Right, Lt.: Left, PAC: plasma aldosterone concentration (pg/mL), C: cortisol ( $\mu \mathrm{g} / \mathrm{dL}), \mathrm{IVC}$ : inferior vena cava, AV: adrenal vein, PAC/C lateralization ratio: $\mathrm{PAC} / \mathrm{C}$ in the right adrenal vein divided by $\mathrm{PAC} / \mathrm{C}$ in the left adrenal vein, $\mathrm{ACTH}$ : adrenocorticotropic hormone, and after ACTH loading: sampling 25 minutes after bolus intravenous injection of $0.25 \mathrm{mg}$ of $\mathrm{ACTH}$. 


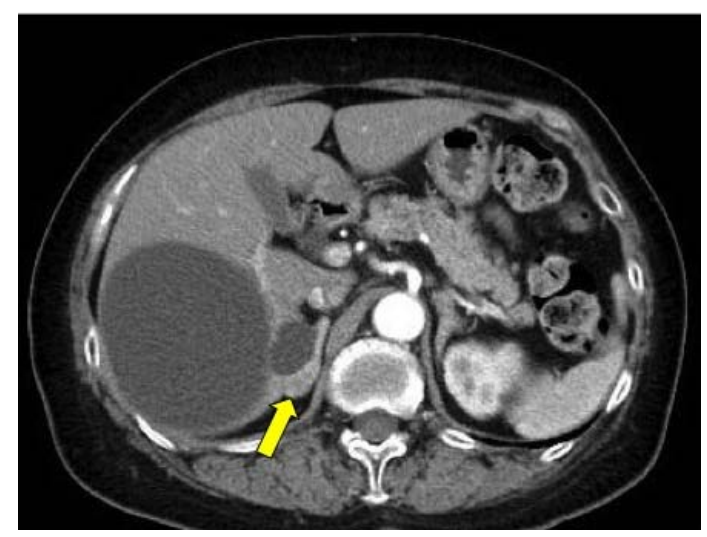

Figure 1. An enhanced computed tomography (CT) scan reveals a right adrenal tumor on the inner side of the liver (arrow), possibly originating from the adrenohepatic fusion.

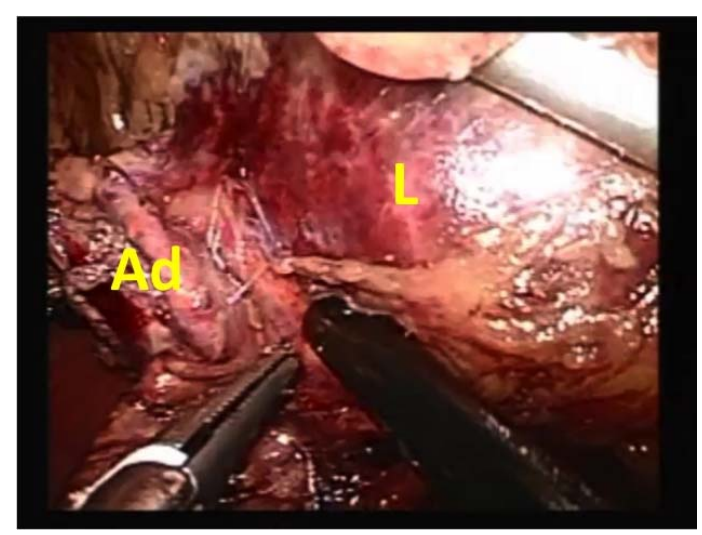

(a)

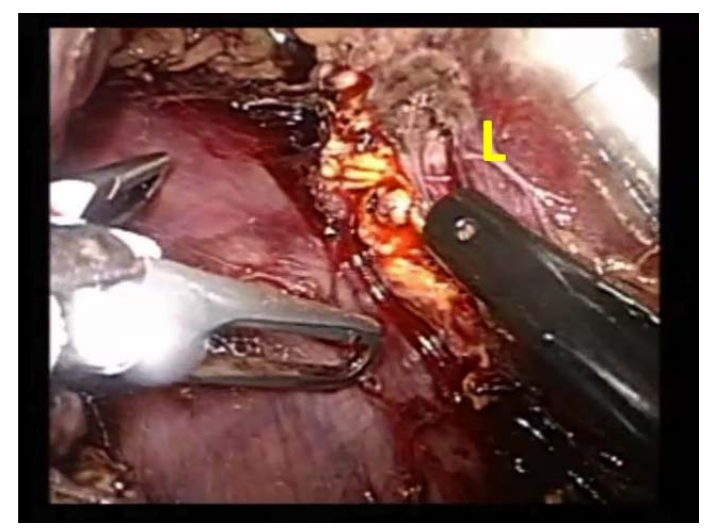

(b)

Figure 2. (a) The right adrenal gland was solidly attached to the liver. L: liver and Ad: right adrenal; (b) The right adrenal gland outside the liver was resected leaving the adrenohepatic fusion tissue inside the liver. L: liver.

sia might present with an adrenocorticotropic hormone (ACTH)-stimulated soft-tissue mass originating from the adrenal rest, particularly in the gonads and retroperitoneum [13]. Functioning and non-functioning adrenal rest tumors of the liver are rarely reported and furthermore,

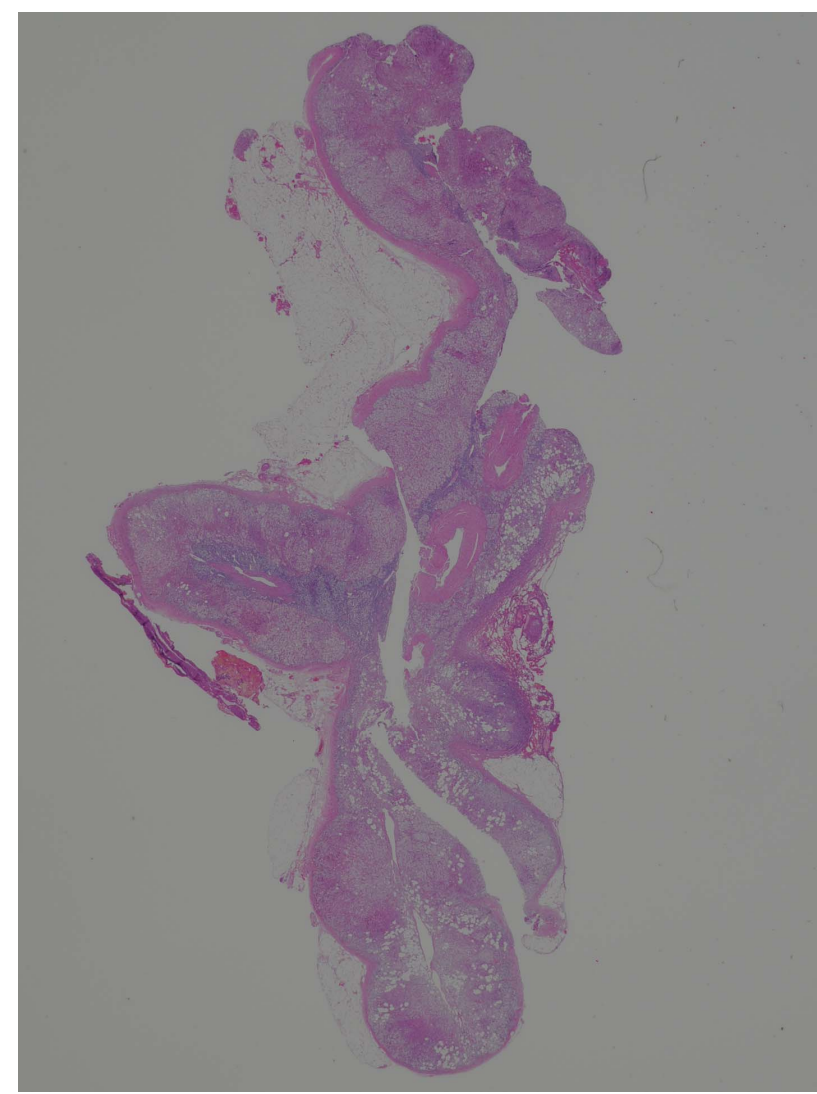

Figure 3. Hematoxylin-eosin staining of the resected adrenal gland, with no adenomas in the tissue.

these tumors are difficult to distinguish from the hepatocellular carcinomas located at the periphery of right hepatic lobe [14-18].

The present case might be a right adrenal tumor originating from the adrenohepatic fusion and cannot be an adrenal rest tumor because the right adrenal gland was not surgically separated from the hepatic parenchyma. In addition, venous sampling from the right adrenal vein revealed elevated aldosterone levels. If the tumor was an adrenal rest tumor that should be isolated from the right adrenal tissue by definition, separating the right adrenal cortex from the liver would have been possible. Moreover, preoperative elevated aldosterone levels would have been detected in the hepatic veins instead of its detection in the right adrenal vein.

Partial hepatectomy might be necessary to surgically treat primary aldosteronism in this patient. However, her blood pressure was well controlled by medical therapy, and she did not prefer hepatectomy to be further performed. Therefore, the medical therapy was continued. However, in this case, it seemed difficult to appropriately diagnose an adrenal tumor originating from the adrenohepatic fusion. It could be diagnosed as a hepatic tumor in some cases and an extrahepatic right adrenal tumor in others. When laparoscopic or open surgery is planned in these 
potentially confusing cases, it might be necessary to consider a possibility of the unexpected intraoperative diagnosis and the immediate measures to be performed based on the diagnosis. According to the medical databases, there are no previous cases of patients where intrahepatic adrenal tumors originating from the adrenohepatic fusion were identified during the laparoscopic or open surgery.

\section{REFERENCES}

[1] G. P. Rossi, "A Comprehensive Review of the Clinical Aspects of Primary Aldosteronism," Nature Reviews Endocrinology, Vol. 7, No. 8, 2011, pp. 485-495. http://dx.doi.org/10.1038/nrendo.2011.76

[2] M. Salvà, M. V. Cicala and F. Mantero, "Primary Aldosteronism: The Role of Confirmatory Tests," Hormone and Metabolic Research, Vol. 44, No. 3, 2012, pp. 177180. http://dx.doi.org/10.1055/s-0032-1304661

[3] P. Mulatero, S. Monticone, C. Bertello, G. Mengozzi, D. Tizzani, A. Iannaccone and F. Veglio, "Confirmatory Tests in the Diagnosis of Primary Aldosteronism," Hormone and Metabolic Research, Vol. 42, No. 6, 2010,pp. 406-410. http://dx.doi.org/10.1055/s-0029-1246186

[4] O. Steichen, F. Zinzindohoué, P. F. Plouin and L. Amar, "Outcomes of Adrenalectomy in Patients with Unilateral Primary Aldosteronism: A Review," Hormone and Metabolic Research, Vol. 44, No. 3, 2012, pp. 221-227. http://dx.doi.org/10.1055/s-0031-1299681

[5] L. H. Honoré and K. E. O’Hara, “Combined Adrenorenal Fusion and Adrenohepatic Adhesion: A Case Report with Review of the Literature and Discussion of Pathogenesis," Journal of Urology, Vol. 115, No. 3, 1976, pp. 323325.

[6] R. Chamanza, H. A. Marxfeld, A. I. Blanco, S. W. Naylor and A. E. Bradley, "Incidences and Range of Spontaneous Findings in Control Cynomolgus Monkeys (Macaca fascicularis) Used in Toxicity Studies," Journal of Toxicologic Pathology, Vol. 38, No. 4, 2010, pp. 642-657. http://dx.doi.org/10.1177/0192623310368981

[7] O. Quesada-Canales, A. Suárez-Bonnet, G. A. Ramírez, M. Aguirre-Sanceledonio, M. Andrada, M. Rivero and A. Espinosa de Los Monteros, "Adrenohepatic Fusion in Domestic Ferrets (Mustela putorius furo),"Journal of Comparative Pathology, Vol. 149, No. 2-3, 2013, pp. 314-317. http://dx.doi.org/10.1016/j.jcpa.2013.02.003

[8] S. Iwamoto, K. Okuda, N. Takeda, K. Sonoda and H. Sanefuji, "Case Report: Right-Sided Periadrenal Metastasis Supplied by the Hepatic Artery. Clue to the Genesis of Pedunculated Hepatocellular Carcinoma," Journal of Gastroenterology and Hepatology, Vol. 12, No. 5, 1997, pp. 392-397.

http://dx.doi.org/10.1111/j.1440-1746.1997.tb00449.x

[9] K. Okuda, M. Arakawa, Y. Kubo, K. Sakata, M. Kage, S.
Iwamoto, S. Takeda, K. Sonoda and H. Sanefuji, "RightSided Pedunculated Hepatocellular Carcinoma: A Form of Adrenal Metastasis," Hepatology, Vol. 27, No. 1, 1998, pp. 81-85. http://dx.doi.org/10.1002/hep.510270114

[10] K. Okano, H. Usuki and H. Maeta, "Adrenal Metastasis from Hepatocellular Carcinoma through an ADRENOHEPATIC Fusion," Journal of Clinical Gastroenterology, Vol. 38, No. 10, 2004, p. 912. http://dx.doi.org/10.1097/00004836-200411000-00019

[11] H. S. Woo, K. H. Lee, S. Y. Park, H. S. Han, C. J. Yoon and Y. H. Kim, "Adrenal Cortical Adenoma in Adrenohepatic Fusion Tissue: A Mimic of Malignant Hepatic Tumor at CT," American Journal of Roentgenology, Vol. 188, No. 3, 2007, pp. W246-W248. http://dx.doi.org/10.2214/AJR.05.0498

[12] B. K. Park, C. K. Kim, B. C. Jung and Y. L. Suh, "Cortical Adenoma in Adrenohepatic Fusion Tissue: Clue to Making a Correct Diagnosis at Preoperative Computed Tomography Examination," European Urology, Vol. 56, No. 6, 2009, pp. 1082-1085.

http://dx.doi.org/10.1016/j.eururo.2009.05.007

[13] T. D. Barwick, A. Malhotra, J. A. Webb, M. O. Savage and R. H. Reznek, "Embryology of the Adrenal Glands and Its Relevance to Diagnostic Imaging," Clinical Radiology, Vol. 60, No. 9, 2005, pp. 953-959. http://dx.doi.org/10.1016/j.crad.2005.04.006

[14] Y. M. Shin, "Hepatic Adrenal Rest Tumor Mimicking Hepatocellular Carcinoma," Korean Journal of Hepatology, Vol. 16, No. 3, 2010, pp. 338-341. http://dx.doi.org/10.3350/kjhep.2010.16.3.338

[15] Y. Baba, T. Beppu, K. Imai, T. Masuda, K. Iyama, H. Sasano and H. Baba, "A Case of Adrenal Rest Tumor of the Liver: Radiological Imaging and Immunohistochemical Study of Steroidogenic Enzymes," Hepatology Research, Vol. 38, No. 11, 2008, pp. 1154-1158. http://dx.doi.org/10.1111/j.1872-034X.2008.00360.x

[16] K. Arai, H. Muro, M. Suzuki, N. Oba, K. Ito and H. Sasano, "Adrenal Rest Tumor of the Liver: A Case Report with Immunohistochemical Investigation of Steroidogenesis," Pathology International, Vol. 50, No. 3, 2000, pp. 244-248. http://dx.doi.org/10.1046/j.1440-1827.2000.01029.x

[17] P. Contreras, E. Altieri, C. Liberman, A. Gac, A. Rojas, A. Ibarra, M. Ravanal, M. Serón-Ferré, “Adrenal Rest Tumor of the Liver Causing Cushing's Syndrome: Therapy with Ketoconazole Preceding an Apparent Surgical Cure," The Journal of Clinical Endocrinology \& Metabolism, Vol. 60, No. 1, 1985, pp. 21-28. http://dx.doi.org/10.1210/jcem-60-1-21

[18] E. Z. Wallace, J. R. Leonidas, A. E. Stanek and A. Avramides, "Endocrine Studies in a Patient with Functioning Adrenal Rest Tumor of the Liver," American Journal of Medicine, Vol. 70, No. 5, 1981, pp. 1122-1125. http://dx.doi.org/10.1016/0002-9343(81)90886-X 